potential of IDH mutations in cancer. Cancer Cell. 2018;34(2):186195.

3. Clark O, Yen K, Mellinghoff IK. Molecular pathways: isocitrate dehydrogenase mutations in cancer. Clin Cancer Res. 2016;22(8):1837-1842.

4. Cancer Genome Atlas Research N, Ley TJ, Miller C, et al. Genomic and epigenomic landscapes of adult de novo acute myeloid leukemia. N Engl J Med. 2013;368(22):2059-2074.

5. Metzeler KH, Herold T, Rothenberg-Thurley M, et al. Spectrum and prognostic relevance of driver gene mutations in acute myeloid leukemia. Blood. 2016;128(5):686-698.

6. Medeiros BC, Fathi AT, DiNardo CD, Pollyea DA, Chan SM, Swords $\mathrm{R}$. Isocitrate dehydrogenase mutations in myeloid malignancies. Leukemia. 2017;31(2):272-281.

7. Ragon BK, DiNardo CD. Targeting IDH1 and IDH2 mutations in acute myeloid leukemia. Curr Hematol Malig Rep. 2017;12(6):537546.

8. Golub D, lyengar N, Dogra S, et al. Mutant isocitrate dehydrogenase inhibitors as targeted cancer therapeutics. Front Oncol. 2019;9:417.

9. Chaturvedi A, Herbst L, Pusch S, et al. Pan-mutant-IDH1 inhibitor BAY1436032 is highly effective against human IDH1 mutant acute myeloid leukemia in vivo. Leukemia. 2017;31(10):2020-2028.

10. Heuser M, Palmisiano N, Mantzaris I, et al. Safety and efficacy of BAY1436032 in IDH1-mutant AML: phase I study results. Leukemia. 2020;34(11):2903-2913

11. Intlekofer AM, Shih AH, Wang B, et al. Acquired resistance to IDH inhibition through trans or cis dimer-interface mutations. Nature. 2018;559(7712):125-129

12. Choe S, Wang H, DiNardo CD, et al. Molecular mechanisms mediating relapse following ivosidenib monotherapy in IDH1-mutant relapsed or refractory AML. Blood Adv. 2020;4(9):1894-1905.

13. Dinardo CD, Stein AS, Stein EM, et al. Mutant IDH (mIDH) inhibitors, ivosidenib or enasidenib, with azacitidine (AZA) in patients with acute myeloid leukemia (AML). J Clin Oncol. 2018;36(15 Suppl):S7042

\title{
Ironing out an approach to alleviate the hypoferremia of acute inflammation
}

\section{Karin E. Finberg}

Yale School of Medicine, New Haven, CT, USA

E-mail: KARIN E. FINBERG - karin.finberg@yale.edu

doi:10.3324/haematol.2020.266627

I $\mathrm{n}$ the steady state, iron levels in the plasma are regulated by the recycling of iron from senescent red blood cells by macrophages of the reticuloendothelial system. In systemic infections and inflammatory states, perturbation of this process can result in hypoferremia (a decrease in circulating iron levels), which may represent a host defense mechanism to limit iron availability to pathogens. ${ }^{1}$ Because hypoferremia restricts the availability of iron to erythroid precursors, if sustained, it contributes to the development of the anemia of inflammation. In this issue of Haematologica, Agoro et al. ${ }^{2}$ report that the acute hypoferremic response to lipopolysaccharide (LPS), a major component of the outer membrane of Gram-negative bacteria, is modulated in mice by pretreatment with a truncated, C-terminal fragment of the hormone fibroblast growth factor 23.

During inflammatory states, iron is sequestered in cells due to a reduction in activity of ferroportin, the major cellular iron exporter that is expressed by multiple cell types, including macrophages. ${ }^{3}$ Activity of the ferroportin transporter on cell membranes is regulated by hepcidin, the key iron regulatory hormone synthesized primarily by the liver; hepcidin occludes the ferroportin transporter and triggers its endocytosis and degradation. ${ }^{4}$ Hepcidin expression is induced in response to several proinflammatory cytokines, including interleukin-6 (IL$6)$ and IL-1 $\beta$, as well as LPS. ${ }^{1}$ In addition to its post-translational regulation by hepcidin, ferroportin expression is regulated at the mRNA level by inflammatory stimuli. In macrophages, stimulation of toll-like receptor 4 (TLR4), a member of the pattern recognition receptor family, with LPS suppresses ferroportin mRNA and protein levels and also induces hepcidin expression. ${ }^{5}$ Stimulation of TLR2 and TLR6 also promotes ferroportin downregulation in a hepcidin-independent manner. ${ }^{6}$ In humans, LPS injection induces an acute rise in plasma cytokines such as IL-6 and tumor necrosis factor (TNF), which is followed by hepcidin elevation, and ultimately a reduction in serum iron levels.?

Recent studies have suggested intriguing crosstalk between inflammation, iron homeostasis, and fibroblast growth factor 23 (FGF23), ${ }^{8}$ a hormone that functions as a key regulator of phosphate and calcium homeostasis. FGF23 in the circulation appears to be mainly derived from bone, although FGF23 expression has also been detected in other tissues. ${ }^{9}$ Physiological actions of FGF23 are mediated through fibroblast growth factor receptors (FGFR) and by the co-receptor Klotho, which increases affinity of FGF23 for FGFR and is required for the hormone's ability to promote renal phosphate excretion. ${ }^{10}$ The phosphaturic activity of the mature, biologicallyactive FGF23 peptide can be abrogated by proteolytic cleavage at an RXXR motif located at the boundary between the FGF core homology domain and the 72amino-acid C-terminal portion of FGF23. ${ }^{9}$ The isolated Cterminal FGF23 fragment (referred to here as C-FGF23) competes with full-length FGF23 for binding to the FGFRKlotho complex, thereby impairing FGF23 signaling; accordingly, in healthy rodents, C-FGF23 administration inhibits renal phosphate excretion and induces hyperphosphatemia. ${ }^{11}$

Circulating FGF23 levels are markedly elevated in chronic kidney disease, ${ }^{9}$ a condition in which disruption of systemic iron homeostasis, mediated by factors such as inflammation, therapy-related iron losses, decreased glomerular filtration rate, low serum erythropoietin (EPO), and elevated serum hepcidin, contributes to the development of anemia. ${ }^{12}$ Both iron deficiency and inflammation stimulate the production of FGF23 as well as its proteolytic cleavage. ${ }^{8}$ Interestingly, in mice with established chronic kidney disease (induced by subtotal nephrectomy), a single dose of C-FGF23 induced acute 
Modulation of LPS responses by C-FGF23 peptide pretreatment

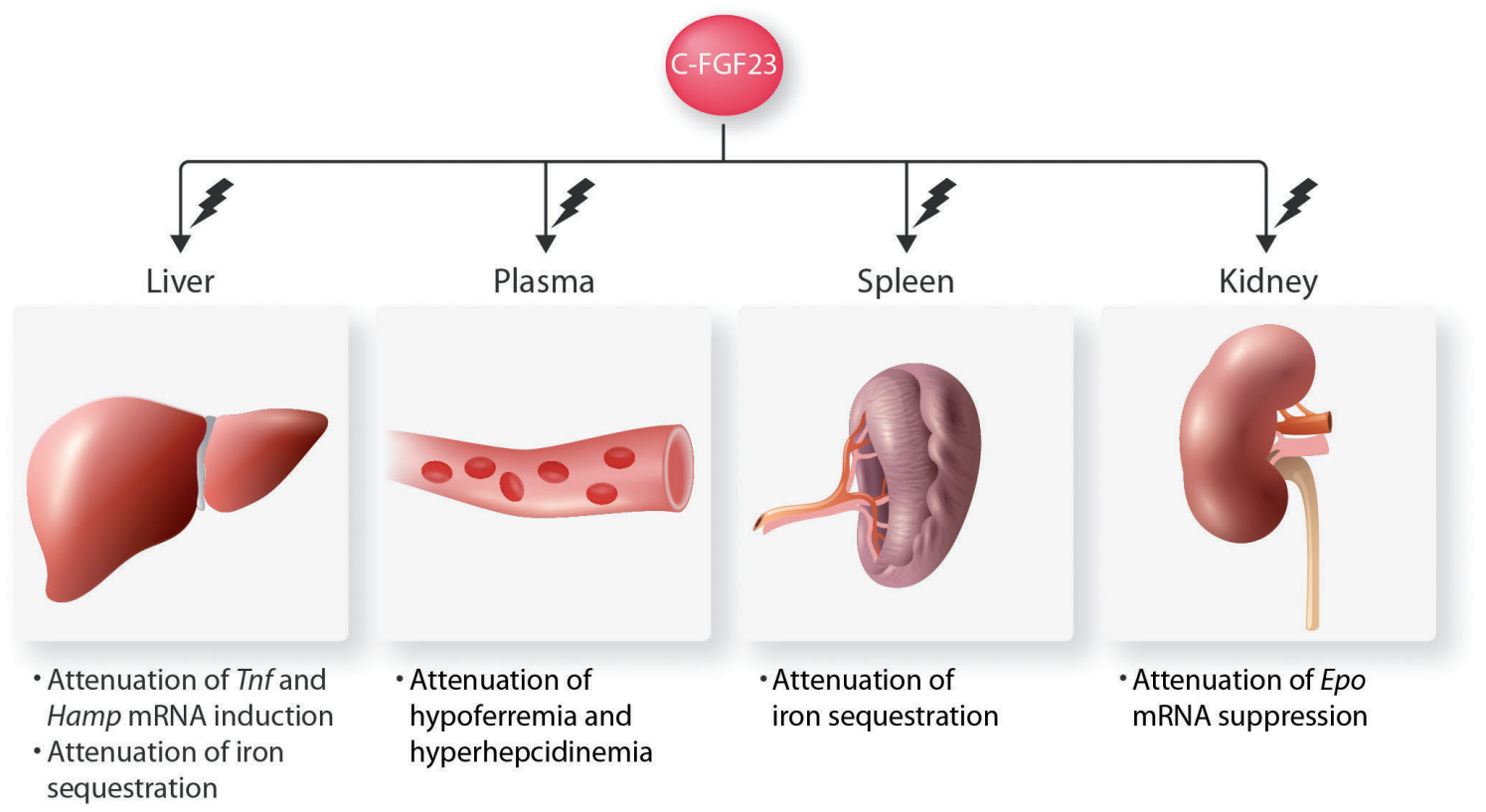

Figure 1. Physiological responses to lipopolysaccharide (LPS), an inducer of acute inflammation, are modulated in mice by pretreatment with the C-terminal portion of the endocrine hormone FGF23 (C-FGF23). The attenuated responses involve parameters that are relevant to systemic iron balance and the development of the anemia of inflammation.

alterations in several physiological parameters relevant to iron homeostasis and erythropoiesis. ${ }^{13}$

In the present study, Agoro et al. ${ }^{2}$ considered the ability of C-FGF23 administration to modulate iron balance in a model of acute systemic inflammation. First, the authors characterized the detailed time course of early physiological responses in wild-type mice subjected to a single, sublethal dose of LPS. As expected, compared to vehicletreated control mice, mice treated with LPS showed transient elevations in hepatic levels of mRNAs encoding proinflammatory cytokines, which peaked one hour after injection. These increases in inflammatory markers were soon followed by anticipated changes in iron-related parameters, including an elevation of hepatic hepcidin mRNA, a reduction in serum iron, a reduction in ferroportin mRNA in liver and spleen, and a rise in splenic iron content. Additionally, compatible with prior studies indicating that FGF23 is regulated by inflammation, transient elevations of Fgf23 mRNA were observed in bone, bone marrow, liver, and spleen, and were accompanied by a transient rise in FGF23 protein in the circulation.

Next, the authors examined the ability of C-FGF23 pretreatment to modulate these acute inflammatory and ironrelated physiological responses in LPS-treated mice. Mice received an intraperitoneal injection of C-FGF23 (or vehicle control) 8 hours prior to challenge with an intraperitoneal injection of LPS (or vehicle control); all mice were analyzed 4 hours after the challenge. LPS-mediated induction of hepatic Tnf and Hamp (hepcidin) mRNA, as well as induction of hyperhepcidinemia, was blunted by C-FGF23 pretreatment (Figure 1). Additionally, C-FGF23 pretreatment attenuated the ability of LPS to induce hypoferremia and to increase tissue iron levels in liver and spleen.
While changes in iron trafficking in response to systemic immune activation contribute significantly to the development of the anemia of inflammation, inflammatory suppression of erythropoietic activity also plays a major role. ${ }^{1}$ Notably, LPS-treatment has been shown to suppress renal Epo mRNA levels in rodents. ${ }^{14}$ Interestingly, Agoro and colleagues found that C-FGF23 pretreatment alleviated LPS-mediated suppression of mRNAs encoding EPO and the EPO receptor in the kidney and also in liver and spleen. C-FGF23 pretreatment also raised serum EPO levels in LPS-treated mice. Additionally, the ability of LPS to induce Fgf23 mRNA in bone and FGF23 protein in the circulation was attenuated by C-FGF23 pretreatment.

By demonstrating a novel link between C-FGF23 and systemic iron modulation in the acute inflammatory setting, the study of Agoro et al. ${ }^{2}$ raises a number of questions to stimulate future investigation. First, what are the physiological mechanisms by which C-FGF23 pretreatment alters LPS-induced hypoferremia and attenuates iron storage in the liver and spleen? Although hepcidin induction by LPS was blunted in mice pretreated with C-FGF23, serum hepcidin levels nevertheless remained markedly elevated compared to control groups. Furthermore, the suppression of hepatic and splenic ferroportin mRNA in response to LPS was not alleviated by C-FGF23 pretreatment. Whether C-FGF23 has direct actions on the duodenum, the major site of dietary iron absorption in the intestine, remains to be explored.

The underlying mechanism by which C-FGF23 modulates LPS-mediated hepcidin induction also requires further clarification. Interestingly, this effect was not explained by a reduction in hepatic expression of IL-6, the most established cytokine regulator of hepcidin in 
response to inflammation, ${ }^{15}$ nor did C-FGF23 pretreatment reduce hepatic levels of phosphorylated STAT3, the major intracellular mediator of hepcidin induction by inflammatory cytokines. ${ }^{1}$ However, interestingly, in livers of LPStreated mice, C-FGF23 pretreatment lowered levels of mRNA encoding BMP6, a key ligand for a major signaling pathway promoting hepcidin transcription. ${ }^{16}$ Additionally, in LPS-treated mice, C-FGF23 pretreatment raised splenic mRNA levels of erythroferrone, a negative regulator of hepcidin expression that is synthesized by erythroblasts. ${ }^{17}$

Another area to be clarified is whether the observed effects of C-FGF23 pretreatment on gene expression, as well as on iron storage, in liver and spleen are mediated through the ability of C-FGF23 to act as a competitive inhibitor of FGFR expressed by these organs. Indeed, although the co-receptor Klotho is expressed primarily in the kidney, parathyroid gland, and brain, recent work suggests that under conditions of FGF23 elevation the FGF23 hormone can participate in FGFR-dependent signaling mechanisms in tissues lacking Klotho. ${ }^{10}$ Another possibility is that C-FGF23 may mediate FGFR-independent biological actions that either directly or indirectly impact iron regulatory pathways. Because the gene expression responses found to be modulated by C-FGF23 were measured in organs of heterogenous cellular composition, such as liver and spleen, it will also be valuable to clarify the participating cell types.

It is also worth considering the fact that the FGF23 Cterminal peptide introduced into mice in this study corresponds to the human peptide sequence; the murine and humans forms of FGF23 share $64 \%$ amino acid homology across this portion of the protein. To exclude the possibility that some observed responses to human C-FGF23 in mice reflect more general immunomodulatory effects that occur due to the introduction of a foreign peptide, it will be valuable to clarify if pretreatment with murine CFGF23 produces similar effects on iron homeostasis in LPS-treated mice.

In terms of clinical relevance, the ability of C-FGF23 to attenuate the hypoferremia, hepatic and splenic iron sequestration, and renal suppression of Epo mRNA observed 4 hours after LPS injection raises the intriguing possibility that C-FGF23-based approaches may have relevance for treatment of anemia of inflammation. Future studies in which the ability of C-FGF23 to modulate these physiological parameters is assessed longitudinally after LPS injection will be informative. Additionally, it will be of great interest to determine if long-term administration of C-FGF23 produces beneficial effects on iron metabolism and erythropoiesis in animal models in which anemia of inflammation has already been achieved through chronic exposure to infectious or noninfectious stimuli. ${ }^{18}$ It will also be of interest to characterize the effects of chronic C-FGF23 administration on phosphate and calcium homeostasis in animal models of the anemia of inflammation.

\section{Disclosures}

No conflicts of interest to disclose.

\section{References}

1. Weiss G, Ganz T, Goodnough LT. Anemia of inflammation. Blood. 2019;133(1):40-50

2. Agoro R, Park MY, Le Henaff C, et al. C-FGF23 peptide alleviates hypoferremia during acute inflammation. Haematologica. 2020;106(2):391-403.

3. Drakesmith H, Nemeth E, Ganz T. Ironing out ferroportin. Cell Metab. 2015;22(5):777-787.

4. Camaschella C, Nai A, Silvestri L. Iron metabolism and iron disorders revisited in the hepcidin era. Haematologica. 2020;105(2):260-272.

5. Peyssonnaux C, Zinkernagel AS, Datta V, Lauth X, Johnson RS, Nizet V. TLR4-dependent hepcidin expression by myeloid cells in response to bacterial pathogens. Blood. 2006;107(9):3727-3732.

6. Guida C, Altamura S, Klein FA, et al. A novel inflammatory pathway mediating rapid hepcidin-independent hypoferremia. Blood. 2015;125(14):2265-2275

7. Kemna E, Pickkers P, Nemeth E, van der Hoeven H, Swinkels D. Time-course analysis of hepcidin, serum iron, and plasma cytokine levels in humans injected with LPS. Blood. 2005;106(5):1864-1866.

8. Babitt JL, Sitara D. Crosstalk between fibroblast growth factor 23, iron, erythropoietin, and inflammation in kidney disease. Curr Opin Nephrol Hypertens. 2019;28(4):304-310.

9. Edmonston D, Wolf M. FGF23 at the crossroads of phosphate, iron economy and erythropoiesis. Nat Rev Nephrol. 2020;16(1):7-19.

10. Richter B, Faul C. FGF23 actions on target tissues-with and without Klotho. Front Endocrinol (Lausanne). 2018;9:189.

11. Goetz R, Nakada Y, Hu MC, et al. Isolated C-terminal tail of FGF23 alleviates hypophosphatemia by inhibiting FGF23-FGFR-Klotho complex formation. Proc Natl Acad Sci U S A. 2010;107(1):407-412.

12. van Swelm RPL, Wetzels JFM, Swinkels DW. The multifaceted role of iron in renal health and disease. Nat Rev Nephrol. 2020;16(2):77-98.

13. Agoro R, Montagna A, Goetz R, et al. Inhibition of fibroblast growth factor 23 (FGF23) signaling rescues renal anemia. FASEB J. 2018;32(7):3752-3764.

14. Frede S, Fandrey J, Pagel H, Hellwig T, Jelkmann W. Erythropoietin gene expression is suppressed after lipopolysaccharide or interleukin-1 beta injections in rats. Am J Physiol. 1997;273(3 Pt 2):R10671071.

15. Nemeth E, Rivera S, Gabayan V, et al. IL-6 mediates hypoferremia of inflammation by inducing the synthesis of the iron regulatory hormone hepcidin. J Clin Invest. 2004;113(9):1271-1276.

16. Wang CY, Babitt JL. Liver iron sensing and body iron homeostasis Blood. 2019;133(1):18-29.

17. Kautz L, Jung G, Valore EV, Rivella S, Nemeth E, Ganz T. Identification of erythroferrone as an erythroid regulator of iron metabolism. Nat Genet. 2014;46(7):678-684.

18. Rivera S, Ganz T. Animal models of anemia of inflammation. Semin Hematol. 2009;46(4):351-357. 